\title{
Lagrange and Wilson theorems for the generalized Stirling numbers
}

\author{
By E. T. BeLL.
}

(Received 11th February, 1938. Read 4th March, 1938.)

1. If $m, n$ are integers, $m>0, n>1$, the generalized Stirling numbers ${ }^{1} S_{r}^{(m)}(n-1)$ are defined by the identity in $x$,

$$
\prod_{a=1}^{n-1}\left(x+a^{m}\right) \equiv \sum_{r=0}^{n-1} S_{r}^{(m)}(n-1) x^{n-r-1} .
$$

The following notation will be fixed.

$p$ is any prime $>0 ; m$ is any integer $>0$.

$\phi(m)$ is the number of positive integers $\leqq m$ and prime to $m$.

$(a, b)$ is the greatest common divisor of the non-negative integers $a, b ;(0, b)=b$ if $b>0$.

$p \equiv \mu \bmod m,(m, \mu)=1,0<\mu \leqq m$.

$(\mu-1, m)=g$.

$(a)_{b}$ is the binomial coefficient $a ! / b !(a-b) !, a>0 ;(a)_{0}=1$.

$S_{r}=S_{r}^{(m)}(p-1)$.

Note that as $\mu$ runs through its $\phi(m)$ values, $p$ runs through all positive primes.

We shall consider the interdependence of the five theorems, $L, F, W, L^{\prime}, W^{\prime}$ :

L. (Lagrange's.) $\prod_{a=1}^{p-1}(x-a) \equiv x^{p-1}-1 \bmod p$, in which $\equiv$ is the sign of identical congruence (the coefficients of like powers of $x$ on both sides are congruent $\bmod p$ ).

$F$. (Fermat's). $\quad x^{p-1}-1 \equiv 0 \bmod p$ has the $p-1$ incongruent roots $1, \ldots, p-1$.

${ }^{1}$ So designated by C. Tweedie, Proceedings Edinburgh Mathematical Society, 37 (1918-19), p. 24. 
W. (Wilson's). $\quad 1+(p-1) ! \equiv 0 \bmod p$.

$L^{\prime} \cdot \prod_{a=1}^{p-1}\left(x-a^{m}\right) \equiv\left(x^{(p-1) / g}-1\right)^{g} \bmod p$.

$W^{\prime} \cdot{ }^{1} \quad S_{r} \equiv 0 \bmod p, 0 \leqq r \leqq p-1, r \neq 0 \bmod (p-1) / g ;$

$S_{t(p-1) / g} \equiv(-1)^{t(p-q-1) / g}(g)_{t} \bmod p, 0 \leqq t \leqq g$.

If only one of these five, say $A$, is used in the deduction of another. say $B$, we shall write $A>B$; if $A>B$ and $B>A$, we write $A=B$. Hence, if $A, B, C$ are any three of the five such that $A>B, B=C$, we can assert $A>C$. Obviously, if $A>B$ and $B>C$, then $A>C$. If none of the five is used in the deduction of $A$, we write $0>A$. In this symbolism we shall prove
$0>L$
(3) $L>F$;
(4) $L>W$;
(5) $L^{\prime}=W^{\prime}$;
(6) $L=L^{\prime}$.

2. As in the usual proofs, (3), (4) are immediate consequences of (2), and (5) is obvious. To recall a proof of (2), we let $n$ in (1) be an odd prime and take $m=1$. In the resulting identity $x$ is replaced by $x+1$, and the new identity is multiplied throughout by $x+1$. Comparison of like powers of $x$ then gives $S_{1}^{(1)}(n-1)=\frac{1}{2} n(n-1)$, $\equiv 0 \bmod n$. From this the successive equations for $S_{r}^{(1)}(n-1), r>1$, give $W^{\prime}$ in the case $p=n, m=1$, and from this $L$ follows for the same $p, m$. Since $L$ holds for $p=2$, the proof of (2) is complete.

Again, (6) is $L>L^{\prime}$ and $L^{\prime}>L$, the second of which follows on taking $m=1$ in $L^{\prime}$. For then $\phi(m)=1$, and $\mu=1$ is the only value of $\mu$, so that $g=1$, and hence $L^{\prime}>L$. We shall give a proof of $L>L^{\prime}$ in $\S 3$.

A shorter proof of $L^{\prime}$, which however is essentially less simple than the proof by $0>L, L>L^{\prime}$, in that it tacitly uses several known theorems which require longer proofs, is as follows. In $L^{\prime}$ replace $1^{m}, \ldots,(p-1)^{m}$, as permissible, by their least positive residues $\bmod p$. Among these residues each of the $(p-1) / g m-i c$ residues of $p$, which are the incongruent roots of $x^{(p-1) / g}-1 \equiv 0 \bmod p$, occurs $g$ times. Hence we have $L^{\prime}$.

3. Let $\theta=e^{2 \pi i / m}$, and in the statement of $L$ replace $x$ by $\theta^{8} x$. A short reduction gives

$$
\prod_{a=1}^{p-1}\left(x-a \theta^{s}\right) \equiv x^{p-1}-\theta^{(p-1) 8} \bmod p
$$

1 The case $m=2$ of $W^{\prime}$ was given by Glaisher, Quarterly Journal, 31 (1900), 34. His method differs from that used here to obtain the general result, and would probably be troublesome to extend. 
In this we take $s=0, \ldots, m-1$ and form the products of corresponding members of the resulting $m$ congruences. Then

$$
\prod_{a=1}^{p-1}\left(x^{m}-a^{m}\right) \equiv \prod_{8=0}^{m}\left(x^{p-1}-\theta^{(p-18)}\right) \bmod p .
$$

Referring to the notation in $\S 1$, we write $p=k m+\mu,(\mu-1, m)=g$, $\mu-1=g \sigma, m=g n,(n, \sigma)=1$. Hence $p-1=g(k n+\sigma)$, and we have

$$
\prod_{a=1}^{p-1}\left(x^{n}-a^{m}\right) \equiv \prod_{8=0}^{g n}\left(x^{(p-1) / g}-e^{28 \sigma \pi i / n}\right) \bmod p .
$$

If $s_{1} \neq s_{2}$ and $s_{1}<n, s_{2}<n$, the congruence $s_{1} \sigma \equiv s_{2} \sigma \bmod n$ is impossible, since $(n, \sigma)=1$. Hence

$$
\prod_{s=0}^{n}\left(x^{(p-1) /(}-e^{28 \sigma \pi i / n}\right)=\left(x^{n(p-1) / g}-1\right)^{g},
$$

and we have

$$
\prod_{a=1}^{p-1}\left(x^{n}-a^{m}\right) \equiv\left(x^{n(p-1) / g}-1\right)^{g} \bmod p .
$$

The last, with $x$ replaced by $x^{1 / n}$, is $L^{\prime}$. Hence $L>L^{\prime}$.

California Institute of Technology,

Pasadena, California, U.S.A. 